\title{
Artificial neural networks for satellite image classification of shoreline extraction for land and water classes of the north west coast of Peninsular Malaysia
}

\begin{abstract}
Monitoring and measuring the shoreline of coastal zones helps establish the boundary of a country. Such an activity entails ground survey, topographic survey, aerial photo, or remote sensing techniques to extract the shoreline. For example, the remote sensing technique to determine shorelines involves the extraction of relevant data from satellite images. Specifically, the satellite image classification enables shorelines to be extracted from land and water classes with a high degree of precision. However, extracting information from satellite images is challenging as it relies on a strong understanding of image processing, machine learning, and data mining techniques. Thus, the researchers discuss the study of the pixel-based classification of machine learning techniques to classify land and water classes in terms of accuracy, training time, and testing time. The research findings showed that the Multilayer Perceptron Artificial Neural Network (MLP ANN) was the most effective technique, compared with other techniques, hence reinforcing its importance in classifying land and water classes.
\end{abstract}

Keyword: Artificial neural networks; Image classification; Machine learning; Multilayer perceptron; Shoreline extraction 